\title{
Information Systems and Rough Set Approximations: An Algebraic Approach
}

\author{
Md. Aquil Khan ${ }^{1}$ and Mohua Banerjee ${ }^{2}$ \\ 1 The Institute of Mathematical Sciences, \\ C.I.T Campus, Chennai 600113, India \\ mdaquilkhan@gmail.com \\ 2 Department of Mathematics and Statistics, \\ Indian Institute of Technology, Kanpur 208016, India \\ mohua@itt.ac.in
}

\begin{abstract}
The article proposes an algebraic formalism of (complete/ incomplete) information systems. Besides providing a complete description of information systems, the formalism captures the notion of rough set approximations that are induced by information systems.
\end{abstract}

\section{Introduction}

Rough set theory proposed by Pawlak involves two key notions: set approximations and information systems. It considers a situation where we only have partial information about a set $U$ of objects, represented by an equivalence relation $R$ on $U$. The pair $(U, R)$ is called an approximation space [8] The relation $R$ induces lower and upper approximation operators $\underline{R}$ and $\bar{R}$ on $\wp(U)$, the power set of $U$, as follows. Let $R(x)$ denote $\{y \in U:(x, y) \in R\}$.

$$
\underline{R}(X):=\{x \in U: R(x) \subseteq U\} \text {, and } \bar{R}(X):=\{x \in U: R(x) \cap X \neq \emptyset\} .
$$

A practical realization of Pawlak's approximation spaces comes from the notion of complete information systems defined as follows.

Definition 1. A complete information system $(C I S) \mathcal{S}:=\left(U, \mathcal{A}, \bigcup_{a \in \mathcal{A}} \mathcal{V}_{a}, f\right)$, comprises a non-empty set $U$ of objects, $\mathcal{A}$ of attributes, $\mathcal{V}_{a}$ of attribute values for each $a \in \mathcal{A}$, and $f: U \times \mathcal{A} \rightarrow \bigcup_{a \in \mathcal{A}} \mathcal{V}_{a}$ such that $f(x, a) \in \mathcal{V}_{a}$

Given a CIS $\mathcal{S}:=\left(U, \mathcal{A}, \bigcup_{a \in \mathcal{A}} \mathcal{V}_{a}, f\right)$, and $B \subseteq \mathcal{A}$, we obtain an approximation space $\left(U, \operatorname{Ind} d_{B}^{S}\right)$, where

$(x, y) \in \operatorname{Ind}_{B}^{\mathcal{S}}$ if and only if $f(x, a)=f(y, a)$, for all $a \in B$.

$\operatorname{Ind}_{B}^{\mathcal{S}}$ is called the indiscernibility relation induced by $B$.

An algebraic approach to rough set theory was first presented by Iwiński in 1987 [6]. Since then, substantial work has been done on algebraic aspects of the theory. In one direction, different representations of rough sets have been considered, and endowed with algebraic structures. It is observed that the algebras induced from approximation spaces are instances of various known as well as new algebraic structures, such as quasi-Boolean algebra, double Stone algebra, Nelson algebra, Łukasiewicz algebra, topological quasi-Boolean algebra. A detailed 
survey can be found in [1]. In the other direction of research, approximation operators are viewed as abstract unary operators, which leads us to a class of Boolean algebra with operators $(\mathrm{BAO})$. In fact, a CIS $\mathcal{S}:=\left(U, \mathcal{A}, \bigcup_{a \in \mathcal{A}} \mathcal{V}_{a}, f\right)$ determines an algebra $\left(\wp(U), \cap, \sim, \emptyset,\left\{\overline{\operatorname{Ind_{B}^{\mathcal {S}}}}\right\}_{B \subseteq \mathcal{A}}\right)$, where $\emptyset$ denotes the empty set, $\sim$, complementation relative to $U$, and $\cap$, intersection. In [4, such an algebra is called a knowledge approximation algebra of type $\mathcal{A}$ derived from $C I S \mathcal{S}$. An abstract algebra for this class of algebras is proposed in [4], and a corresponding representation theorem obtained.

We note that the knowledge approximation algebra derived from a CIS $\mathcal{S}$ does not give a complete description of the CIS. In fact, attribute, attribute-value pairs, which are the main ingredients of a CIS, do not appear in this description. In this article, our aim is to propose an algebraic formalism of CISs which captures this aspect. Moreover, it also captures the notion of approximations defined on CISs. In Sect. 2, we shall consider an algebra induced by CISs. An abstract algebra is then proposed, of which such an induced algebra is an instance. Properties of the proposed abstract algebra are explored. Sect. 3 provides the corresponding representation theorem. Sect. 4 concludes the article.

\section{Algebra for Complete Information Systems}

Let us fix finite sets $\mathcal{A}$ of attributes and $\mathcal{V}:=\bigcup_{a \in \mathcal{A}} \mathcal{V}_{a}$ of attribute values. Let $\mathcal{D}$ denote the set of all descriptors [9], viz. pairs $(a, v)$, for each $a \in \mathcal{A}$, $v \in \mathcal{V}_{a}$. Observe that, given a complete information system $\mathcal{S}:=(U, \mathcal{A}, \mathcal{V}, f)$, each descriptor $(a, v)$ determines a nullary operation (constant) $c_{(a, v)}^{\mathcal{S}}$ on $\wp(U)$ :

$$
c_{(a, v)}^{\mathcal{S}}:=\{x \in U: f(x, a)=v\} .
$$

Thus we have the following definition. Let $\Omega$ be the tuple $(\mathcal{A}, \mathcal{V})$.

Definition 2. Let $\mathcal{S}:=(U, \mathcal{A}, \mathcal{V}, f)$ be a complete information system. A complete information system algebra (in brief, CIS-algebra) of type $\Omega$ generated by the complete information system $\mathcal{S}$ is the structure

$$
\mathcal{S}^{*}:=\left(\wp(U), \cap, \sim, \emptyset,\left\{\underline{\operatorname{Ind}_{B}^{\mathcal{S}}}\right\}_{B \subseteq \mathcal{A}},\left\{c_{\gamma}^{\mathcal{S}}\right\}_{\gamma \in \mathcal{D}}\right) .
$$

Observe that a CIS-algebra generated by a CIS $\mathcal{S}$ is actually an extension of the knowledge approximation algebra derived from $\mathcal{S}$ with a collection of nullary operations.

One can show that a CIS-algebra satisfies the properties listed below.

Proposition 1. 1. $\bigcup_{v \in \mathcal{V}_{a}} c_{(a, v)}^{\mathcal{S}}=U$.

2. $c_{(a, v)}^{\mathcal{S}} \cap c_{(a, u)}^{\mathcal{S}}=\emptyset$ when $v \neq u$.

3. $\underline{\operatorname{Ind} d_{C}^{\mathcal{S}}}(X) \subseteq \underline{\operatorname{Ind}} \mathcal{S}_{B}^{\mathcal{S}}(X)$ for $C \subseteq B \subseteq \mathcal{A}$, and $X \subseteq U$.

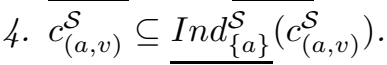

5. $c_{(b, v)}^{\mathcal{S}} \cap \overline{\operatorname{Ind}_{B \cup\{b\}}^{\mathcal{S}}}(X) \subseteq \underline{\operatorname{Ind}_{B}^{\mathcal{S}}}\left(\sim c_{(b, v)}^{\mathcal{S}} \cup X\right), X \subseteq U$.

6. $\operatorname{Ind}_{\emptyset}^{\mathcal{S}}(X) \neq \emptyset$ implies $X=U$. 
We shall see later that these properties are actually characterizing properties of CIS-algebras. Thus, we propose the following notion of an abstract CIS-algebra.

Definition 3. An abstract CIS-algebra of type $\Omega$ is a tuple

$$
\mathfrak{A}:=\left(U, \wedge, \neg, 0,\left\{L_{B}\right\}_{B \subseteq \mathcal{A}},\left\{d_{\gamma}\right\}_{\gamma \in \mathcal{D}}\right),
$$

where $(U, \wedge, \neg, 0)$ is a Boolean algebra and $L_{B}$ and $d_{\gamma}$ are respectively unary and nullary (constant) operations on $U$ satisfying the following:

$\left(C_{1}\right) \bigvee_{v \in \mathcal{V}_{a}} d_{(a, v)}=1$;

$\left(C_{2}\right) d_{(a, v)} \wedge d_{(a, u)}=0$ when $v \neq u$;

$\left(C_{3}\right) L_{C}(x) \leq L_{B}(x)$ for $C \subseteq B \subseteq \mathcal{A}$;

$\left(C_{4}\right) d_{(a, v)} \leq L_{\{a\}}\left(d_{(a, v)}\right)$;

$\left(C_{5}\right) d_{(b, v)} \wedge L_{B \cup\{b\}}(x) \leq L_{B}\left(\neg d_{(b, v)} \vee x\right)$;

$\left(C_{6}\right) L_{\emptyset}(x) \neq 0$ implies $x=1$.

As a consequence of Proposition 1, the CIS-algebra $\mathcal{S}^{*}$ generated by a CIS $\mathcal{S}$ is an abstract CIS-algebra.

Let $U_{B}$ be the dual of the operator $L_{B}$, that is, $U_{B}(x):=\neg L_{B}(\neg x)$. The following proposition presents a few properties of abstract CIS-algebras.

Proposition 2. 1. $U_{B}(0)=L_{B}(0)=0$ and $U_{B}(1)=L_{B}(1)=1$.

2. $L_{B}(x) \leq x \leq U_{B}(x)$.

3. $U_{B}\left(x \wedge U_{B} y\right)=U_{B}(x) \wedge U_{B}(y)$ and $L_{B}\left(x \vee L_{B} y\right)=L_{B}(x) \vee L_{B}(y)$.

4. $x \neq 0$ implies $U_{\emptyset} x=1$.

5. $U_{B}\left(U_{B}(x)\right)=U_{B}(x)$ and $L_{B}\left(L_{B}(x)\right)=L_{B}(x)$.

6. $U_{B}(x \vee y)=U_{B}(x) \vee U_{B} y$ and $L_{B}(x \wedge y)=L_{B}(x) \wedge L_{B} y$.

From Proposition 2, it is clear that $U_{B}$ and $L_{B}$ are respectively closure and interior operators. Moreover, the reduct $\mathfrak{A}:=\left(U, \wedge, \neg, 0,\left\{L_{B}\right\}_{B \subseteq \mathcal{A}}\right)$ is a topological Boolean algebra [1]. Furthermore, $\left(U, \wedge, \neg, 0,\left\{U_{B}\right\}_{B \subseteq \mathcal{A}}\right)$ satisfies all the conditions of abstract knowledge approximation algebra 4] except the following. In the latter case, the reduct $(U, \wedge, \neg, 0)$ is taken to be a complete atomic Boolean algebra, while we do not have that requirement. An abstract knowledge approximation algebra also needs to satisfy $U_{B \cup C}(x)=U_{B}(x) \wedge U_{C}(x), x$ being an atom, and this, in general, may not hold in an abstract CIS-algebra.

Let us recall that a cylindric algebra of dimension $|\mathcal{A}|[5]$ is a structure $\mathfrak{A}:=$ $\left(U, \wedge, \neg, 0,\left\{\Lambda_{a}\right\}_{a \in \mathcal{A}},\left\{\mu_{(a, b)}\right\}_{(a, b) \in \mathcal{A} \times \mathcal{A})}\right.$, where $(U, \wedge, \neg, 0)$ is a Boolean algebra, and $\Lambda_{a}, \mu_{(a, b)}$ are respectively unary and nullary operations on $U$, such that

$\left(L_{1}\right) \Lambda_{a}(0)=0,\left(L_{2}\right) x \leq \Lambda_{a}(x),\left(L_{3}\right) \Lambda_{a}\left(x \wedge \Lambda_{a} y\right)=\Lambda_{a}(x) \wedge \Lambda_{a}(y)$,

$\left(L_{4}\right) \Lambda_{a}\left(\Lambda_{b}(x)\right)=\Lambda_{b}\left(\Lambda_{a}(x)\right),\left(L_{5}\right) \mu_{(a, a)}=1$,

$\left(L_{6}\right)$ If $a \neq b, c$, then $\mu_{(b, c)}=\Lambda_{a}\left(\mu_{(b, a)} \wedge \mu_{(a, c)}\right)$,

$\left(L_{7}\right)$ If $a \neq b$, then $\Lambda_{a}\left(\mu_{(a, b)} \wedge x\right) \wedge \Lambda_{a}\left(\mu_{(a, b)} \wedge \neg x\right)=0$.

The difference between the signature of an abstract CIS-algebra of type $(\mathcal{A}, \mathcal{V})$, and that of a cylindric algebra of dimension $|\mathcal{A}|$ is now clear. The cylindric algebra has unary and nullary operations corresponding to each element of $\mathcal{A}$, and $\mathcal{A} \times \mathcal{A}$ respectively. Whereas, in the case of abstract CIS-algebra, unary 
and nullary operations are indexed respectively over the sets $\wp(\mathcal{A})$ and $\mathcal{A} \times \mathcal{V}$. Moreover, operators $U_{B}$ of an abstract CIS-algebra satisfy $\left(L_{1}\right)-\left(L_{3}\right)$, but may fail to satisfy $\left(L_{4}\right)$. $\left(L_{5}\right)-\left(L_{7}\right)$ do not make sense in the case of abstract CISalgebras. However, the $\mathrm{BAO}\left(U, \wedge, \neg, 0, U_{B}\right)$ obtained from an abstract CISalgebra is a cylindric algebra of dimension 1 .

\section{Representation}

In this section, we shall prove the representation theorem for abstract CISalgebras. Here, we would like to mention that the proof of the representation theorem for abstract knowledge approximation algebras given in 4] makes use of the completeness and atomicity properties of the Boolean algebra reduct of the algebra. In fact, the embedding of an abstract knowledge approximation algebra $\mathfrak{A}$ is given in an extension of the power set algebra over the set $A t(\mathfrak{A})$ of atoms of $\mathfrak{A}$. But in the case of abstract CIS-algebras, the Boolean algebra reduct may not be complete and atomic, and hence this technique will not work. We use prime filters [3] for our purpose.

Recall that a filter of a Boolean algebra $\mathfrak{A}:=(U, \wedge, \sim, 0)$ is a subset $F$ of $U$ such that (i) $1 \in F$, (ii) if $a, b \in F$, then $a \wedge b \in F$, (iii) if $a \in F$ and $a \leq b$, then $b \in F$. A filter is proper if it does not contain the smallest element 0 . A proper filter is prime if $a \vee b \in F$ implies that at least one of $a$ and $b$ belongs to $F$.

Let $\operatorname{PF}(\mathfrak{A})$ denote the set of all prime filters of $\mathfrak{A}$.

Let us consider an abstract CIS-algebra $\mathfrak{A}:=\left(U, \wedge, \neg, 0,\left\{L_{B}\right\}_{B \subseteq \mathcal{A}},\left\{d_{\alpha}\right\}_{\alpha \in \mathcal{D}}\right)$. $\mathfrak{A}$ determines a unique CIS $\mathfrak{A}_{*}$ as follows.

Consider the mapping $f_{\mathfrak{A}}: P F(\mathfrak{A}) \times \mathcal{A} \rightarrow \mathcal{V}$ such that

$$
f_{\mathfrak{A}}(\Gamma, a)=v \text { if and only if } d_{(a, v)} \in \Gamma \text {. }
$$

Conditions $\left(C_{1}\right)$ and $\left(C_{2}\right)$ in Definition 3 guarantee that $f_{\mathfrak{A}}$ is a total function. Thus, we obtain the CIS $\mathfrak{A}_{*}:=\left(P F(\mathfrak{A}), \mathcal{A}, \mathcal{V}, f_{\mathfrak{A}}\right)$. $\mathfrak{A}_{*}$ determines the lower approximation operators $\operatorname{Ind}_{B}^{\mathfrak{A}_{*}}, B \subseteq \mathcal{A}$, on $\wp(P F(\mathfrak{A}))$.

We also recall that the reduct $\left(U, \wedge, \neg, 0,\left\{L_{B}\right\}_{B \subseteq \mathcal{A}}\right)$ of an abstract CISalgebra $\mathfrak{A}:=\left(U, \wedge, \neg, 0,\left\{L_{B}\right\}_{B \subseteq \mathcal{A}},\left\{d_{\alpha}\right\}_{\alpha \in \mathcal{D}}\right)$ determines a complex algebra [3] as follows.

For each $B \subseteq \mathcal{A}$, let us consider the binary relation $Q_{B}^{\mathfrak{A}} \subseteq P F(\mathfrak{A}) \times P F(\mathfrak{A})$ defined as follows:

$$
(\Gamma, \Delta) \in Q_{B}^{\mathfrak{A}} \text { if and only if } L_{B}(x) \in \Gamma \text { implies } x \in \Delta .
$$

The relations $Q_{B}^{\mathfrak{A}}$ are used to define the operators $m_{B}^{\mathfrak{A}}: \wp(P F(\mathfrak{A})) \rightarrow \wp(P F(\mathfrak{A}))$ :

$$
m_{B}^{\mathfrak{A}}(X):=\left\{\Gamma \in P F(\mathfrak{A}): \text { for all } \Delta \text { such that }(\Gamma, \Delta) \in Q_{B}^{\mathfrak{A}}, \Delta \in X\right\} .
$$

The complex algebra corresponding to the reduct $\left(U, \wedge, \neg, 0,\left\{L_{B}\right\}_{B \subseteq \mathcal{A}}\right)$ of the abstract CIS-algebra $\mathfrak{A}$ is given by extending the power set algebra over $P F(\mathfrak{A})$ with the operators $m_{B}^{\mathfrak{A}}$.

So, an abstract CIS-algebra $\mathfrak{A}$, on the one hand, determines the lower approximation operators $\operatorname{Ind} d_{B}^{\mathfrak{A}_{*}}$. On the other hand, it gives rise to the complex algebra with operators $\overline{m_{B}^{\mathfrak{A}} \text {. Is }}$ there any relationship between the operators $m_{B}^{\mathfrak{A}}$, and the lower approximation operators $\operatorname{In} d_{B}^{\mathfrak{A} *}$ ? In fact, we shall now show that 
for each $B \subseteq \mathcal{A}$, the operators $m_{B}^{\mathfrak{A}}$ and $\operatorname{In} d_{B}^{\mathfrak{A}_{*}}$ are the same. This result will also lead us to the desired representation theorem. Let us begin with the following proposition listing a few properties of the relations $Q_{B}^{\mathfrak{A}}$.

Proposition 3. 1. $Q_{B}^{\mathfrak{A}} \subseteq Q_{C}^{\mathfrak{A}}$ for $C \subseteq B \subseteq \mathcal{A}$.

2. $d_{(b, v)} \in \Gamma \cap \Delta$ for some $v \in \mathcal{V}_{b}$ if and only if $(\Gamma, \Delta) \in Q_{\{b\}}^{\mathfrak{A}}$.

3. If $(\Gamma, \Delta) \in Q_{B}^{\mathfrak{A}}$ and $d_{(b, v)} \in \Gamma \cap \Delta$ for some $v \in \mathcal{V}_{b}$, then $(\Gamma, \Delta) \in Q_{B \cup\{b\}}^{\mathfrak{A}}$.

4. $Q_{\emptyset}^{\mathfrak{A}}=P F(\mathfrak{A}) \times P F(\mathfrak{A})$.

5. $Q_{B}^{\mathfrak{A}}=\bigcap_{b \in B} Q_{\{b\}}^{\mathfrak{A}}$.

Proof. (11) is a direct consequence of $\left(C_{3}\right)$. Let us prove (2). First suppose $d_{(b, v)} \in$ $\Gamma \cap \Delta$ for some $v \in \mathcal{V}_{b}$, and let $L_{\{b\}}(x) \in \Gamma$. We need to show $x \in \Delta$. Using the properties of filters, we obtain $d_{(b, v)} \wedge L_{\{b\}}(x) \in \Gamma$ and hence by $\left(C_{5}\right)$ with $B=\emptyset$, we obtain $L_{\emptyset}\left(\neg d_{(b, v)} \vee x\right) \in \Gamma$. This shows that $L_{\emptyset}\left(\neg d_{(b, v)} \vee x\right) \neq 0$ and hence by $\left(C_{6}\right)$, we obtain $\neg d_{(b, v)} \vee x=1$. Therefore, we have $\neg d_{(b, v)} \vee x \in \Delta$. Finally using the fact that $d_{(b, v)} \in \Delta$, we obtain $x \in \Delta$.

Conversely, suppose $(\Gamma, \Delta) \in Q_{\{b\}}^{\mathfrak{A}}$. By $\left(C_{1}\right)$, there exists a $v \in \mathcal{V}_{b}$ such that $d_{(b, v)} \in \Gamma$. Therefore, using $\left(C_{4}\right)$, we obtain $L_{\{b\}}\left(d_{(b, v)}\right) \in \Gamma$, and so $d_{(b, v)} \in \Delta$.

Let us now prove (3). Suppose $(\Gamma, \Delta) \in Q_{B}^{\mathfrak{A}}$ and $d_{(b, v)} \in \Gamma \cap \Delta$ for some $v \in \mathcal{V}_{b}$. Further suppose $L_{B \cup\{b\}}(x) \in \Gamma$. We need to show $x \in \Delta$. Due to the given conditions, we obtain $d_{(b, v)} \wedge L_{B \cup\{b\}}(x) \in \Gamma$, and hence by $\left(C_{5}\right)$, $L_{B}\left(\neg d_{(b, v)} \vee x\right) \in \Gamma$. This gives $\neg d_{(b, v)} \vee x \in \Delta$, as $(\Gamma, \Delta) \in Q_{B}^{\mathfrak{A}}$. As $d_{(b, v)} \in \Delta$, $x \in \Delta$.

(41) is obvious due to $\left(C_{6}\right)$. Let us now move to (5). From (11), we obtain $Q_{B}^{\mathfrak{A}} \subseteq \bigcap_{b \in B} Q_{\{b\}}^{\mathfrak{A}}$. It is also not difficult to see that the reverse inclusion holds when $|B| \leq 1$. To complete the proof, let us assume that the reverse inclusion holds for $B$, and prove it for $B \cup\{a\}$. Let $(\Gamma, \Delta) \in \bigcap_{b \in B \cup\{a\}} Q_{\{b\}}^{\mathfrak{A}}$. We need to show $(\Gamma, \Delta) \in Q_{B \cup\{a\}}^{\mathfrak{A}}$. Using (2) and the fact that $(\Gamma, \Delta) \in Q_{\{a\}}^{\mathfrak{A}}$, we obtain $d_{(a, v)} \in \Gamma \cap \Delta$ for some $v$. Now (3) gives $(\Gamma, \Delta) \in Q_{B \cup\{a\}}^{\mathfrak{A}}$.

As a consequence of Proposition 3, we obtain

Theorem 1. Let $\mathfrak{A}:=\left(U, \wedge, \neg, 0,\left\{L_{B}\right\}_{B \subseteq \mathcal{A}},\left\{d_{\gamma}\right\}_{\gamma \in \mathcal{D}}\right)$ be an abstract CISalgebra. Then for each $B \subseteq \mathcal{A}$, Ind $d_{B}^{\mathfrak{A}_{*}}=Q_{B}^{\mathfrak{A}^{-}}$and $\underline{\operatorname{Ind}_{B}^{\mathfrak{A}_{*}}}=m_{B}^{\mathfrak{A}}$.

Theorem 2 (Representation theorem for abstract CIS-algebras).

Let $\mathfrak{A}:=\left(U, \wedge, \neg, 0,\left\{L_{B}\right\}_{B \subseteq \mathcal{A}},\left\{d_{\gamma}\right\}_{\gamma \in \mathcal{D}}\right)$ be an abstract CIS-algebra. Then the mapping $\Psi: U \rightarrow \wp(P F(\mathfrak{A}))$ given by

$$
\Psi(x):=\{\Gamma \in P F(\mathfrak{A}): x \in \Gamma\}, x \in U,
$$

is an embedding of $\mathfrak{A}$ into $\left(\mathfrak{A}_{*}\right)^{*}$.

Proof. It is not difficult to see that $\Psi\left(d_{\gamma}\right)=c_{\gamma}^{\mathfrak{A}_{*}}, \gamma \in \mathcal{D}$. Due to Theorem 1, the rest follows in the lines of the proof of Jóhnson-Tarski theorem (cf. [3]).

Let us consider a language $\mathcal{L}$ consisting of a countable set $\operatorname{Var}:=\{p, q, r, \ldots\}$ of variables, a binary operator $\wedge$, unary operators $\neg, \mathbf{L}_{B}$ and constants $\mathbf{0}, \mathbf{d}_{(a, v)}$, 
where $B \subseteq \mathcal{A},(a, v) \in \mathcal{D}$. The well-formed formulae (wffs) of $\mathcal{L}$ are defined recursively: $\alpha:=p \in \operatorname{Var}|\mathbf{0}| \mathbf{d}_{(a, v)}|\neg \alpha| \alpha \wedge \beta \mid \mathbf{L}_{B} \alpha$.

Now consider an abstract CIS-algebra $\mathfrak{A}:=\left(U, \wedge, \neg, 0,\left\{L_{B}\right\}_{B \subseteq \mathcal{A}},\left\{d_{\gamma}\right\}_{\gamma \in \mathcal{D}}\right)$. An assignment for $\mathfrak{A}$, is a map $V: \operatorname{Var} \rightarrow U$. $V$ can be extended to a mapping $\tilde{V}$ from the set of all $\mathcal{L}$-wffs to $U$ in the obvious way: $\mathbf{0}, \boldsymbol{d}_{(a, v)}, \boldsymbol{L}_{B}$ correspond respectively to $0, d_{(a, v)}, L_{B}$. An equation $\alpha \approx \beta$ is said to hold in $\mathfrak{A}$, denoted as $\mathfrak{A}=\alpha \approx \beta$, if $\tilde{V}(\alpha)=\tilde{V}(\beta)$ for all $V$.

The notion of equivalence defined above can be used to realize certain interesting laws related to CISs and approximations. For instance, one may easily verify that $\left(\neg\left(\boldsymbol{d}_{(b, v)} \wedge \boldsymbol{L}_{B \cup\{b\}}(x)\right) \vee\left(\boldsymbol{L}_{B}\left(\neg \boldsymbol{d}_{(b, v)} \vee x\right)\right)\right) \approx \mathbf{1}$ holds in all abstract CIS-algebras. The representation theorem also leads to the complete axiomatization for the semantic notion of equivalence in CIS-algebras generated by CISs. More formally speaking, using Birkhoff's completeness theorem for equational logic [2], one can prove that if $\alpha \approx \beta$ holds in all CIS-algebras generated by CISs, then $\alpha \approx \beta$ is derivable from the equations $\left(C_{1}\right)-\left(C_{6}\right)$. One can also show using the above representation theorem that abstract CIS-algebras form the algebraic counterpart of the logic LIS of complete information systems discussed in 7].

\section{Conclusions}

In this article we have proposed an algebraic formalism of complete information systems. This formalism can be extended to incomplete information systems in a natural way to capture approximations with respect to indiscernibility as well as similarity relations. It would be interesting to see if a similar approach could lead to algebras for non-deterministic information systems.

\section{References}

1. Banerjee, M., Chakraborty, M.K.: Algebras from rough sets. In: Pal, S.K., Polkowski, L., Skowron, A. (eds.) Rough-neuro Computing: Techniques for Computing with Words, pp. 157-184. Springer, Berlin (2004)

2. Birkhoff, G.: On the structure of abstract algebras. In: Proceedings of the Cambridge Philosophical Society, vol. 29, pp. 441-464 (1935)

3. Blackburn, P., de Rijke, M., Venema, Y.: Modal Logic. Cambridge University Press, Cambridge (2001)

4. Comer, S.: An algebraic approach to the approximation of information. Fundamenta Informaticae XIV, 492-502 (1991)

5. Henkin, L., Monk, J.D., Tarski, A.: Cylindric Algebras, Part I. North-Holland Pub. Co., Amsterdam (1971)

6. Iwiński, T.B.: Algebraic approach to rough sets. Bulletin of the Polish Academy of Sciences (Math) 35(9-10), 673-683 (1987)

7. Khan, M.A., Banerjee, M.: A logic for complete information systems. In: Sossai, C., Chemello, G. (eds.) ECSQARU 2009. LNCS, vol. 5590, pp. 829-840. Springer, Heidelberg (2009)

8. Pawlak, Z.: Rough sets. International Journal of Computer and Information Science 11(5), 341-356 (1982)

9. Pawlak, Z.: Rough Sets. Theoretical Aspects of Reasoning about Data. Kluwer Academic Publishers, Dordrecht (1991) 